\title{
CHRONIQUE DE LA RECHERCHE
}

\section{TRICENTENAIRE «BERKELEY » * (1685-1985)}

Le tricentenaire de la naissance de Berkeley (1685-1753) a donné lieu à des commémorations diverses qui intéressent aussi bien l'histoire générale des idées que l'histoire des sciences.

C'est surtout par l'immatérialisme et par la formule « exister c'est être perçu " que le philosophe irlandais nous reste célèbre. Mais il suffit de rappeler son expédition en Amérique, pour le salut des âmes et la conversion des Indiens, ou d'évoquer sa conviction tardive d'une toute-puissance thérapeutique de l'eau de goudron pour le salut des corps, pour que soient mis à jour des intérêts polymorphes. Intérêts polymorphes mais cohérents : Berkeley voyait dans le progrès des sciences la résistible ascension de la libre pensée. Or, l'ensemble des colloques et publications, indiqués ci-après, manifestent une attention de plus en plus soutenue pour l'articulation des préoccupations philosophiques et apologétiques de Berkeley avec un état des sciences de son temps. Et que l'on nous permette de souligner l'importance et la qualité des contributions françaises à ce que les célébrations de 1985 ont apporté à la culture universelle.

- Colloque à Newport, Rhode-Island, 8-10 mars 1985, organisé par l'International Berkeley Society et la Browne University. Actes du colloque publiés sous la direction d'Ernest Sosa : Essays on the Philosophy of George Berkeley, Dordrecht, Reidel, 1985.

- Colloque à Dublin, Trinity College, 19-22 août 1985. Actes publiés sous la direction de David Berman : George Berkeley, Essays and Replies, Hermathena, Dublin, 1985.

- Colloque à Oxford, 26-28 septembre 1985, organisé à la Maison Française sous la direction de Geneviève Brykman. Actes partiellement publiés sous la forme d'un numéro spécial « George Berkeley, 1685-1985 ", History of European Ideas, vol. 7, 6, 1986.

\footnotetext{
- Étant donné l'ampleur des manifestations auxquelles a donné lieu le tricentenaire, le Comité de rédaction a demandé à Geneviève Brykman, auteur d'une importante thèse sur Berkeley, de bien vouloir faire, pour la Revue, un panorama de cette année particulièrement féconde pour l'avenir des études berkeleyennes.
} 
- Journée «Berkeley et les sciences de son temps ", le 22 novembre 1985 à l'Université de technologie de Compiègne.

- Conférence de Geneviève Brykman à la Société française de philosophie, le 23 novembre 1985 : « Passivité des esprits et passivité des idées dans l'immatérialisme de Berkeley. "

- Deux numéros spéciaux " Berkeley ":

Revue internationale de philosophie, fasc. 3, 1985 ;

Revue philosophique, 3, 1986 ; ce dernier numéro contient, entre autres la traduction de $A$ Proposal for the Better Supplying of Churches in our Foreign Plantations, and for Converting the Savage Americans to Christianity, by a College to Be Erected in [...] the Islands of Bermuda (trad. : Roselyne Guérineau).

- Publication d'une édition diplomatique du manuscrit de l'Introduction des Principes par B. Belfrage (Lund University) : George Berkeley's Manuscript Introduction, Oxford, Doxa, 1987.

Pour clore cette floraison de célébrations, annonçons la nouvelle traduction des œuvres de Berkeley en français, sous la direction de Geneviève Brykman :

Berkeley, Euvres, I et II, Paris, P.U.F. ("Épiméthée ").

Ces deux volumes offrent, à l'intérieur de l'œuvre de Berkeley, une sélection des ouvrages proprement philosophiques, dont le critère est donné dans une Introduction générale à partir d'une question que l'évolution du sens des mots impose : que faut-il entendre aujourd'hui par philosophie, dans une œuvre si diverse ?

Le volume I (1985) regroupe les Notes philosophiques (traduction collective) et l'Introduction manuscrite au Traité des Principes de la connaissance humaine (trad. Dominique Berlioz-Letellier) - deux textes décisifs pour comprendre la genèse de l'immatérialisme à l'intérieur d'un projet apologétique plus ambitieux - , ainsi que. l'Essai pour une nouvelle théorie de la vision (trad. Laurent Déchery) et le Traité des Principes (trad. Marilène Phillips).

Le volume II (1987) comporte les Trois dialogues entre Hylas et Philonous (trad. Jean-Marie Beyssade), !a Correspondance avec.Samuel Johnson (trad. Geneviève Brykman), la Nouvelle théorie de la vision défendue et expliquée, enfin deux opuscules qui présentent une importance toute particulière pour l'historien des sciences : $\mathrm{Du}$ mouvement (trad. Michelle Beyssade et Dominique Berlioz), et L'Analyste ou dissertation adressée à un mathématicien infidèle (trad. Michel Blay), augmenté d'une Défense de la libre pensée en mathématiques (trad. Dominique Berlioz).

Outre l'Avant-propos et l'Introduction générale par Geneviève Brykman, chacun des deux volumes contient des présentations brèves et purement informatives de chaque texte par les traducteurs, une chronologie de la vie de Berkeley, un Index thématique et un Index des auteurs. Les notes sont réduites au minimum dans le dessein de ne pas doubler le texte de Berkeley d'un commentaire. Seul L'Analyste échappe à cette règle : l'ouvrage est, cette fois, assorti de notes historiques abondantes dues à Michel Blay : il convenait de faire connaître au lecteur la toile de fond de L'Analyste et un état, souvent mal connu, des controverses sur les méthodes infinitésimales qui fut profitable à la clarification des notions de limite et de continuité. 
Le tricentenaire "Berkeley " aura donc été, en France en particulier, le point de départ f'un renouveau des études berkeleyennes. Comme le philosophe de l'immatérialisme, non seulement interrogeait les savants de son temps sur le bien fondé de leur usage des mots, mais encore avait maille à partir avec les théologiens sur la question des noms divins et sur le pouvoir temporel des églises, on peut dire que l'œuvre de Berkeley apporte une lumière singulière sur son temps.

Geneviève BryKMAN, Professeur à l'Université de Paris-X. 